WM-94-106

\title{
Leading-Log Effects in the Resonance Electroweak Form Factors
}

\author{
Carl E. Carlson ${ }^{(a)}$ and Nimai C. Mukhopadhyay ${ }^{(b)}$ \\ ${ }^{(a)}$ Physics Department, College of William and Mary, Williamsburg, VA 23187 \\ (b) Department of Physics, Rensselaer Polytechnic Institute, Troy, NY 12180-3590
}

(July 12, 2018)

\begin{abstract}
We study $\log$ corrections to inelastic scattering at high Bjorken $x$ for $Q^{2}$ from 1 to $21 \mathrm{GeV}^{2}$. At issue is the presence of log corrections, which can be absent if high $x$ scattering has damped gluon radiation. We find logarithmic correction of the scaling curve extrapolated to low $Q^{2}$ improves the duality between it and the resonance plus background data in the $\Delta$ region, indicating $\log$ corrections exist in the data. However, at $W>2 \mathrm{GeV}$ and high $x$, the data shows a $(1-x)^{3}$ form. Log corrections in one situation but not in another can be reconciled by a $W$ - or $Q^{2}$ - dependent higher twist correction.
\end{abstract}

In this Letter, we investigate possible logarithmic corrections to the inelastic structure function $\nu W_{2}$ at high Bjorken $x$.

As a vehicle we use Bloom-Gilman (BG) duality, which is a relationship [1] 通 between resonance physics and the physics of the deep inelastic region. Bloom and Gilman [1] observed that the ratio of the area underneath a resonance bump in inelastic electron scattering to that of the continuum beneath the bump was generally constant with increasing $Q^{2}$ and that the smooth scaling curve seen at high $Q^{2}$ was an accurate average over the resonance bumps seen at lower $Q^{2}$, but the same Bjorken $x$. The first of these observations appears to be untrue [4] for the $\Delta(1232)$, although for other resonances it is well confirmed out to high 
momentum transfer. The second observation appears to be true in general. In particular, for the $\Delta(1232)$, the background seems to rise [4], as the resonance falls, so that the average is constant relative to the scaling curve. Theoretically, one can understand in a perturbative QCD context [2, 3] that the $Q^{2}$ falloff of the resonance and of the scaling curve evaluated at the $x$ value pertinent to the resonance are the same, at least as far as the powers of $Q^{2}$ are concerned. The dependence on logarithms of $Q^{2}$ has not yet been considered in this context.

In this Letter, we first investigate the logarithmic corrections to the resonance-continuum (or BG) duality discussed above. We compare the resonance data [5,6] to the scaling curve, for $Q^{2}$ from 1 to $21 \mathrm{GeV}^{2}$. We correct the scaling curve using the Altarelli-Parisi evolution equation [7]. We find that the corrections are sizeable and that they improve the duality between the resonance data and the scaling curve.

We proceed by calculating the logarithmic corrections to the predicted resonance form factors using common baryon distribution amplitudes [8]. The logarithmic corrections that ensue do not track those of the scaling curve. We should not necessarily conclude that the BG duality is violated by the logarithmic corrections, having found otherwise from the data. Rather, this suggests a need for better models of the baryon distribution amplitudes, or possibly that some other effect is mimicking the $\log Q^{2}$ behavior.

An issue is whether the logarithmic corrections to the quark distributions coming from the Altarelli-Parisi equation are actually present in the $x \rightarrow 1$ region relevant to low $W$ (hadronic c.m. energy). Brodsky et al. [9] have argued that the gluon radiation that yields the splitting function, and the resultant logarithmic corrections are absent in this region. The criterion for the absence of the gluon radiation is that $(1-x) Q^{2}<\mu^{2}$, where $\mu^{2}$ is some scale on the order of $1 \mathrm{GeV}^{2}$ but is not precisely known. We also note that $(1-x) Q^{2}=$ $x\left(W^{2}-m_{N}^{2}\right) \approx\left(W^{2}-m_{N}^{2}\right)$ (for $\left.x \rightarrow 1\right)$, so that the presence of log corrections in the resonance region is an open question until $\mu^{2}$ is determined.

The measured values of $\nu W_{2}$ [5] at high $x$ (above 0.7 ) and for $W$ above the resonance region $(W>2 \mathrm{GeV})$ have a $(1-x)^{3}$ form. No logarithmic corrections appear to be needed. This is consistent with the prediction of Brodsky et al. [9], but inconsistent with our obser- 
vations in the $\Delta$ region. However, the apparent absence of a logarithmic correction at high $W$ and its importance in the resonance region can be reconciled by a $W$-dependent higher twist correction.

Logs in the continuum for $x \rightarrow 1$. We wish to see what effect logarithmic corrections have on the parton distribution functions, hence on the continuum scaling function. Since the resonance region draws closer to $x=1$ with increasing $Q^{2}$, we shall limit our considerations for the continuum also to $x \rightarrow 1$, and then shall be able to quote some results in analytic form.

We start with the Altarelli-Parisi equation having unsuppressed gluon radiation,

$$
\frac{d q(x, t)}{d t}=\frac{\alpha_{s}(t)}{2 \pi} \int_{0}^{1} d y d z \delta(x-y z) q(y, t) P_{q q}(z)
$$

Here, $t=\ln \left(Q^{2} / \Lambda^{2}\right), \alpha_{s}(t)=4 \pi / \beta_{1} t, \beta_{1}=11-(2 / 3) n_{f}$, (where $n_{f}$ is the number of fermion flavors), and $q(x, t)$ is a quark distribution function of a given flavor. The gluon term is omitted, as its contribution is subleading in $(1-x)$.

The splitting function is

$$
P_{q q}(z)=C_{F}\left\{\frac{1+z^{2}}{(1-z)_{+}}+\frac{3}{2} \delta(1-z)\right\},
$$

where $C_{F}$ is $4 / 3$, and $(1-z)_{+}$is defined by

$$
\int_{x}^{1} d z \frac{f(z)}{(1-z)_{+}}=\int_{x}^{1} d z \frac{f(z)}{1-z}-\int_{0}^{1} d z \frac{f(1)}{1-z}
$$

We want to examine the evolution of a form like $q\left(x, t_{0}\right)=N_{0}(1-x)^{b}$, where $b$ is a constant, and $t_{0}$ corresponds to some benchmark $Q_{0}^{2}$. Hence we use the Ansatz:

$$
q(x, t)=N(x, t)(1-x)^{b}
$$

in the Altarelli-Parisi equation. Systematically throwing away terms of higher order in $(1-x)$,we get

$$
q(x, t)=N_{0}(1-x)^{b+4 C_{F}\left(\ln \ln Q^{2}\right) / \beta_{1}},
$$

where 


$$
\ln \ln Q^{2} \equiv \ln \left(\frac{\ln Q^{2} / \Lambda^{2}}{\ln Q_{0}^{2} / \Lambda^{2}}\right) \equiv T(Q) .
$$

To see the size of the logarithmic correction, we examine the values of $x$ corresponding to the peak of the $\Delta$ resonance region,

$$
\frac{1}{x}=1+\frac{m_{\Delta}^{2}-m_{N}^{2}}{Q^{2}} .
$$

In Table 』, we show the size of the correction factor to the high- $x$ continuum in the $\Delta$-region using $Q^{2}$ of $4 \mathrm{GeV}^{2}$ as a benchmark. The logs are important for the resonance excitation even though the correction to the exponent is fairly mild. For example, the $(1-x)^{3}$ we use for $F_{2}$ at $Q^{2}$ of $4 \mathrm{GeV}^{2}$ is modified by radiative corrections to about $(1-x)^{3.16}$ at $Q^{2} \approx 20$ $\mathrm{GeV}^{2}$. However, the value of $(1-x)$ is very small for the $\Delta$ at the latter $Q^{2}$ leading to a change in $F_{2}$ by a factor $(0.029)^{0.16} \approx 0.57$.

We close this section with two observations. One concerns a parameterization of the quark distribution by Morfin and Tung [10]. It is of the form

$$
q(x, t) \propto(1-x)^{C_{0}+C_{1} T(Q)+C_{2} T^{2}(Q)},
$$

for $x \rightarrow 1$. Here the coefficient $C_{1}$ is just what is here calculated to be $\left(4 C_{F} / \beta_{1}\right)$. For their DIS-scheme fits, we note, they get $C_{1}=0.53-0.54$, whereas

$$
\frac{4 C_{F}}{\beta_{1}}=0.59,0.64,
$$

for the three or four flavors respectively. Our second remark concerns the comparison of $F_{2}=\nu W_{2}$ data in the resonance region to the continuum scaling curve. This is shown in Fig. [1, with and without the $\log$ correction. In each case, the ratio $R=I / S$ is plotted versus $Q^{2}$. Here we have defined

$$
\begin{aligned}
& I=\int_{\Delta \xi} d \xi F_{2}\left(\xi, Q^{2}\right), \\
& S=\int_{\Delta \xi} d \xi F_{2}^{\text {scaling }}\left(\xi, Q^{2}\right),
\end{aligned}
$$


where $\xi$ is the Nachtmann variable ( $x$ corrected 4,12 for the target mass effects), and $\Delta \xi$ is a bite covering the chosen resonance region (here, the $\Delta$ ). Although the error bars are large at high $Q^{2}$, one observes that the logarithmic corrections improve the constancy of the ratio $R$ at high $Q^{2}$. Hence the inclusion of the logarithmic effects helps to make the duality idea, the low- $Q^{2}$ structure function for a given $W$ should average to the scaling curve, appear to work better.

Log $Q^{2}$ effects on the resonance to continuum ratio. The resonance contribution to the inelastic structure function is

$$
F_{2}^{R}=G_{+}^{2}\left(Q^{2}\right) \frac{m_{N}^{2} \Gamma_{R} /\left(2 \pi m_{R}\right)}{\left(W-m_{R}\right)^{2}+\Gamma_{R}^{2} / 4},
$$

assuming a simple Breit-Wigner form, and dropping the sub-leading helicity form factors $G_{0}$ and $G_{-}$. The form factor is

$$
G_{+}\left(Q^{2}\right)=g_{+} \frac{\alpha_{S}^{2}\left(Q^{2}\right)}{Q^{3}} \sum\left(E_{i j} N_{i}^{P} N_{j}^{R}\right)\left(\ln Q^{2}\right)^{-\gamma_{i}^{P}-\gamma_{j}^{R}}
$$

where $N_{P}^{i}$ and $N_{R}^{j}$ are coefficients from the distribution amplitudes of the proton and resonance respectively. The latter is, for example, given by

$$
\Phi^{R}\left(x, Q^{2}\right)=x_{1} x_{2} x_{3} \sum_{i} N_{i}^{R} \tilde{\Phi}_{i}(x)\left(\ln Q^{2}\right)^{-\gamma_{i}}
$$

The $\Phi^{i}$ are Appel polynomials and the anomalous dimensions $\gamma^{i}$ are known and positive. We take the form of the continuum for $x \rightarrow 1$ as

$$
F_{2}^{\text {scaling }}\left(x, Q^{2}\right)=\operatorname{const}(1-x)^{b+C_{1} T}
$$

We compare the resonance and continuum contribution to the ratio of integrals $R=I_{i} / S_{i}$. First,

$$
S_{i}=\int_{\Delta x_{i}} d x F_{2}^{\text {scaling }} \approx \frac{\text { const }}{Q^{8}}\left(\ln Q^{2}\right)^{-C_{1} \ln Q^{2}},
$$

for $b=3$. The other integral is 


$$
\begin{aligned}
I_{i} & =\int_{\Delta x_{i}} d x F_{2}^{R} \approx \frac{\text { const }}{Q^{2}}\left|G_{+}\right|^{2} \\
& \approx \frac{\text { const }}{Q^{8}}\left|\sum_{i j}\left(E_{i j} N_{i}^{P} N_{j}^{R}\right)\left(\ln Q^{2}\right)^{-2-\gamma_{i}^{P}-\gamma_{j}^{R}}\right|^{2},
\end{aligned}
$$

where we have recalled $\alpha_{s} \sim 1 / \ln Q^{2}$.

It is clear that $I_{i}$ and $S_{i}$ have the same power law falloff (for $F_{2}$ scaling $\sim(1-x)^{3}$ for $x \rightarrow 1$ ). The $\ln Q^{2}$ dependences can be approximately the same only under special circumstances and a limited range of the $Q^{2}$. Let us consider only a small range of $\ln Q^{2}$ $\left(\equiv \ln \left(Q^{2} / \Lambda^{2}\right) / \ln \left(Q_{0}^{2} / \Lambda^{2}\right)\right)$ and expand around $\ln Q^{2}=\ln Q_{0}^{2}$, so that $\ln Q^{2}=1+\epsilon$. Then equating the $\mathrm{O}(1)$ and $\mathrm{O}(\epsilon)$ terms of the expansion leads to the relation

$$
\frac{1}{2} C_{1}=\frac{\sum_{i j}\left(E_{i j} N_{i}^{P} N_{j}^{R}\right)\left(2+\gamma_{i}^{P}+\gamma_{j}^{R}\right)}{\sum_{i j}\left(E_{i j} N_{i}^{P} N_{j}^{R}\right)} .
$$

Since $C_{1} / 2$ is about $1 / 4$ and the $\gamma_{i}$ are positive, only exceptional choices of the amplitudes $N_{i}^{P}$ and/or $N_{j}^{R}$ can fulfill the above equation. We know no cases of practical that satisfy Eqn. 17. For instance, it is not fulfilled for the cases [8] of the Chernyak-Zhitnitsky or the King-Sachrajda wave function for the proton, and analogous wave functions for the $\mathrm{S}_{11}(1535)$ or the $\Delta(1232)$. It happens that every significant $E_{i j} N_{i}^{P} N_{j}^{S_{11}}$ is positive, violating Eq.(17). Thus, in general, the BG duality in the form of the constancy of the resonance peak to scaling curve ratio must be logarithmically violated at high $Q^{2}$. The resonance will fall faster than the background. The main reason for this is the $\left(\alpha_{s}\left(Q^{2}\right)\right)^{2}$ factor in the exclusive state form factor, absent for the inclusive process. This quantity falls by a factor of nearly two $\left[1.85\right.$ for $\left.\Lambda_{Q C D}=200 \mathrm{MeV}\right]$, as $Q^{2}$ changes from 4 to $21 \mathrm{GeV}^{2}$. That $Q^{3} G_{+}\left(Q^{2}\right)$ is nearly constant in this range of $Q^{2}$ is interesting and not understood at this level.

The phenomenological parton distribution functions. For our purposes, we need the parton distribution functions for $x \rightarrow 1$. However,the existing parameterizations [10,13 are fit to data at lower $x$, and thus are not designed to be outside specified ranges of $x$. For example, both Morfin and Tung [10] and Botts et al. (the CTEQ collaboration) [11] state their fits to be valid for $x<0.75$; similar restrictions apply to other parameterizations. 
Hence, some diffidence is required in extrapolating these functions toward $x=1$, and one should not be surprised by disagreements among various parameterizations, and between any of them and the data, as $x \rightarrow 1$.

Fig. 2 shows some high $x$, non-resonance region data [5]. The parameterizations of Morfin and Tung [10] and CTEQ [11] are also shown. They fall too rapidly in this region and are below the data by a factor of roughly two at the highest $x$ data point. The naive, uncorrected $(1-x)^{3}$ curve matches the data better. We should note that the logarithmic corrections will not give as dramatic an effect here as in the resonance region. For the example of the points in Fig. 2, where $Q^{2}$ is about $20 \mathrm{GeV}^{2}$, a radiative correction factor of $(1-x)^{0.16}$ falls from 0.83 to 0.74 as we go from the lefthand data point to the right hand data point. That is a change of barely over 10\%, although including it worsens the agreement with the data.

Here we recall the Brodsky et al. [9] argument that the logarithms are healed (absent) for kinematics where $(1-x) Q^{2}$ is small. The high $x$ non-resonance region data does favor the Brodsky et al. suggestion. However, we already have seen the importance of the the QCD radiative corrections in the $\Delta$ region. The two seemingly disparate observations could be reconciled by allowing a $W$-dependent higher twist correction, so that we have in the high $x$ region

$$
F_{2} \propto(1-x)^{3+4 C_{F} T(Q) / \beta_{1}} \times\left(1+C_{2} \frac{m_{N}^{2}}{W^{2}}\right)
$$

where $C_{2}=1.7$ gives the dashed curve in Fig. 2 .

Concluding remarks. We have studied here the leading log QCD radiative corrections at high $Q^{2}$ in and near the resonance region. The region of the $\Delta(1232)$ resonance, in which the resonance bump falls faster than the underlying background, is of special interest. We have found the logarithmic corrections to be important for $Q^{2}>4 \mathrm{GeV}^{2}$. The agreement between the $F_{2}$ data in the resonance region, smoothed over the resonance width, and the scaling curve is much improved by the logarithmic corrections. This indicates that the gluonic radiative corrections are important even in the resonance region. 
We have also considered the effect of the log corrections to the baryon form factors directly. In general, these effects are dependent on the specific baryon wave functions. Log corrections to form factors, for commonly used baryon distribution amplitudes [8], disagree with those to the scaling curve. Accepting the common distribution amplitudes means that the resonance-background duality is violated logarithmically, contradicting the observations summarized in Fig. 1. This suggests the need for better model baryon distribution amplitudes.

The measured $F_{2}=\nu W_{2}$ at high $x$ and $W>2 \mathrm{GeV}$ is nicely fit by a plain $(1-x)^{3}$ form. This agrees with the expectation of Brodsky et al. [9], although $(1-x) Q^{2} \approx 3 \mathrm{GeV}^{2}$ for this region, which is large for the absence of gluonic radiation. The apparent absence of a log correction here is also surprising in the light of its apparent presence in the resonance region, but these can be reconciled by a higher twist correction of the form $\left(1+\right.$ const $\left.m_{N}^{2} / W^{2}\right)$.

One could entertain an alternative explanation of our observations: Log corrections coming from gluonic radiation are absent everywhere in the high $x$ regions we have studied, and a $Q^{2}$ dependent higher twist correction is giving the effect we have observed for the $\Delta$ region. In either case, higher twist corrections are indicated, with different kinematic dependence.

Our conclusions invite more precise and complete experimental tests. In particular, the hypothesis on evolution healing [9] can be tested against the full leading log corrected structure function ameliorated by higher twist corrections discussed here, by measuring that structure function over a range of high $x$ at fixed values of $W$ [14] in one instance and at fixed $Q^{2}$ in another.

Acknowledgments. We thank S. Brodsky and P. Stoler for helpful comments. The research of CEC is supported in part by the NSF (Grant PHY-9306141), and that of NCM by the U.S. Department of Energy (Grant DE-FG02-88ER40448.A006). 


\section{REFERENCES}

[1] E.D. Bloom and F.J. Gilman, Phys. Rev. Lett. 25 (1970) 1140; Phys. Rev. D 4 (1971) 2901.

[2] A. DeRújula, H. Georgi, and H. D. Politzer, Ann. Phys. (N.Y.) 103 (1977) 315.

[3] C.E. Carlson and N.C. Mukhopadhyay, Phys. Rev. D 41 (1990) R2343.

[4] C.E. Carlson and N.C.Mukhopadhyay, Phys. Rev. D 47 (1993) R1737.

[5] P. Stoler, Phys. Rev. Lett. 66 (1991) 1003; Phys. Rev. D 44 (1991) 73; Phys. Rep. 226, 103 (1993); and private communication.

[6] P.E. Bosted et al., Phys. Rev. D 49, 3091 (1994); C. Koeppel, Ph.D. thesis (American University, 1994).

[7] G.Altarelli and G. Parisi, Nucl. Phys. B 126, 298 (1977); see also L. N. Lipatov, Yad. Fiz. 20, 181 (1974) [Sov. J. Nucl. Phys. 20, 94 (1975)] and L. Gribov, E. Levin, and M. Ryskin, Phys. Rep. 100, 1 (1983).

[8] V.L. Chernyak and A.R. Zhitnitsky, Nucl. Phys. B 246 (1984) 52; J.D. King and C.T. Sachrajda, Nucl. Phys. B 279 (1987) 785; C.E. Carlson and J.L. Poor, Phys. Rev. D 38, 2758 (1988); G.R.Farrar, H. Zhang, A.A. Oglublin, and I.R. Zhitnitsky, Nucl. Phys. B 311, 585 (1989); J. Bonekamp, Bonn report(1989).

[9] S.J. Brodsky, Tao Huang, and G.P. Lepage, in Particles and Fields 2 (proceedings of the Banff Summer Institute on Particles and Fields 1981), A.Z. Capri and A.N. Kamal, eds. (Plenum, New York, 1982), p. 143 and S.J. Brodsky, private communication.

[10] J.G. Morfin and W.K. Tung, Z. Phys. C 52 (1991) 13.

[11] J. Botts et al., Phys. Lett. B 304, 159 (1993).

[12] O. Nachtmann, Nucl. Phys. B 63 (1973) 237. 
[13] E.J. Eichten, I. Hinchliffe, and C. Quigg, Phys. Rev. D 45 (1992) 2269.

[14] For example, fixing $W$ at $2 \mathrm{GeV}^{2}$ and letting $x$ vary from 0.85 to 0.75 changes the radiative correction factor, roughly $(1-x)^{0.6 T(Q)}$, by about $20 \%$. The corresponding $Q^{2}$ are 18 and $9.4 \mathrm{GeV}^{2}$, respectively. 


\section{TABLES}

TABLE I. Numerical values for the correction to $(1-x)^{b}$ for the $\Delta(1232)$ excitation kinematics. The function $T(Q)$ is defined in the text. We used $\Lambda=150 \mathrm{MeV}$.

\begin{tabular}{ccc}
\hline \hline$Q^{2} \mathrm{GeV}^{2}$ & $x$ & $(1-x)^{4 C_{F} T(Q) / \beta_{1}}$ \\
\hline 4 & 0.863 & 1.000 \\
6 & 0.904 & 0.901 \\
8 & 0.926 & 0.824 \\
10 & 0.940 & 0.761 \\
12 & 0.950 & 0.711 \\
17 & 0.964 & 0.615 \\
21 & 0.971 & 0.560 \\
\hline \hline
\end{tabular}




\section{FIGURES}

FIG. 1. The effect of logarithmic corrections upon the duality ratio $R$, defined in the text. The heavy circles with uncertainty bars indicate $R$ with log corrections made for the scaling curve; the open circles indicate where central values lie when no log corrections are made (percentage uncertainties are the same). The ratios with corrections lie more closely on a horizontal line, as as predicted by perturbative QCD, drawn here with arbitrary ordinate. 
FIG. 2. Measured $F_{2}=\nu W_{2}$ [5] at high $x$ above the resonance region $(W>2 \mathrm{GeV})$, represented by triangles. The solid line is $(1-x)^{3}$, the tight dashed line is the parameterization DIS of Morfin and Tung [10], the dash-triple-dotted line (close to the Morfin-Tung line) is from the CTEQ1L distribution [11], and the loose dashed line (close to the solid line) is a result including both logarithmic corrections and a $W$ dependent higher twist correction, as described in the text. Values of $Q^{2}$ range from 16 to $19 \mathrm{GeV}^{2}$ for the data in this figure, and values of $W$ range from 2.8 to 2.0 $\mathrm{GeV}$. Uncertainties in the data are about $\pm 10 \%$, or about the size of the triangles. 